\title{
The role of estrogens and estrogen receptor signaling pathways in cancer and infertility: the case of schistosomes
}

\author{
Mónica C. Botelho ${ }^{1,2}$, Helena Alves ${ }^{1}$, Alberto Barros ${ }^{3,4}$, Gabriel Rinaldi ${ }^{5}$, \\ Paul J. Brindley ${ }^{5}$, and Mário Sousa ${ }^{6}$ \\ ${ }^{1}$ INSA, National Institute of Health Dr. Ricardo Jorge, Porto, Portugal \\ ${ }^{2}$ IPATIMUP, Institute of Pathology and Molecular Immunology of the University of Porto, Portugal \\ ${ }^{3}$ Centre for Reproductive Genetics Prof. Alberto Barros, Porto, Portugal \\ ${ }^{4}$ Department of Genetics, Faculty of Medicine, University of Porto, Porto, Portugal \\ ${ }^{5}$ Department of Microbiology, Immunology and Tropical Medicine, and Research Center for Neglected Tropical and Infectious \\ Diseases, School of Medicine \& Health Sciences, George Washington University, Washington, DC 20037, USA \\ ${ }^{6}$ Department of Microscopy, Laboratory of Cell Biology, Institute of Biomedical Sciences Abel Salazar (ICBAS), Multidisciplinary \\ Unit for Biomedical Research-UMIB, University of Porto, Porto, Portugal
}

\begin{abstract}
Schistosoma haematobium, a parasitic flatworm that infects more than 100 million people, mostly in the developing world, is the causative agent of urogenital schistosomiasis, and is associated with a high incidence of squamous cell carcinoma (SCC) of the bladder. Schistosomiasis haematobia also appears to negatively influence fertility, and is particularly associated with female infertility. Given that estrogens and estrogen receptors are key players in human reproduction, we speculate that schistosome estrogen-like molecules may contribute to infertility through hormonal imbalances. Here, we review recent findings on the role of estrogens and estrogen receptors on both carcinogenesis and infertility associated with urogenital schistosomiasis and discuss the basic hormonal mechanisms that might be common in cancer and infertility.
\end{abstract}

The case of schistosomiasis

Schistosomiasis is a neglected tropical disease transmitted to humans from freshwater snails. It is caused by a blood fluke of the genus Schistosoma. Schistosomiasis is considered the most important of the helminthiases and the second most important parasitosis, after malaria, causing high rates of morbidity and mortality. Schistosomes affect at least 76 countries and 200 million people worldwide. From these, 20 million have severe disease and 120 million are considered symptomatic. Risk of infection affects 600 million others including travelers from developed countries [1].

\footnotetext{
Corresponding author: Botelho, M.C. (monicabotelho@hotmail.com, monica.botelho@insa.min-saude.pt).

Keywords: schistosomiasis; estrogen receptor; cancer; infertility.

This opinion focuses on estrogen metabolism and estrogen receptor (ER) signaling pathways associated with cancer induction and female infertility in the context of Schistosoma haematobium infection. The present work attempts to integrate a variety of studies and experimental approaches with $S$. haematobium models, while giving particular emphasis to the in vitro studies that have contributed to expanding our understanding of the mechanisms of action of estrogen metabolism and ER signaling pathways associated with schistosomiasis. In particular, we suggest that hormonal imbalance resulting from $S$. haematobium may promote cancer and infertility.

\section{Urogenital schistosomiasis}

Three major species of schistosomes are the agents of human schistosomiasis - Schistosoma japonicum and Schistosoma mansoni cause intestinal schistosomiasis in East Asia, Africa, South America and the Caribbean, while $S$. haematobium, occurring widely throughout Africa and the Middle East, causes urogenital schistosomiasis. Recent recalibration of health burdens revealed that in the range of 4.5-70 million disability adjusted life years (DALYs) are lost to schistosomiasis. More people are infected with $S$. haematobium than with the other schistosomes combined. Of $\sim 112$ million cases of S. haematobium infection in sub-Saharan Africa, 70 million are associated with hematuria, 18 million with major bladder wall pathology, and 10 million with hydronephrosis leading to kidney damage [2-4]. In many patients, deposition of $S$. haematobium parasite ova eventually leads to squamous cell carcinoma (SCC) of the bladder [5,6]. Accordingly, S. haematobium has been classified as a Group 1 carcinogen by the International Agency for Research on Cancer (IARC) $[7,8]$. In addition, as many as $75 \%$ of women infected with S. haematobium suffer from female genital schistosomiasis (FGS) of the lower genital tract [3]. FGS results from 
deposition of schistosome eggs in the uterus, cervix, vagina, and/or vulva, with ensuing inflammatory responses; it also increases susceptibility of the woman to HIV [9-11]. The resulting FGS is associated with contact bleeding, discharge, pain on intercourse, as well as diminished fertility, besides being a source of shame and stigma [12].

The cellular and molecular mechanisms linking $S$. haematobium infection either with both cancer induction and female infertility remain to be deciphered $[12,13]$. However, estrogen-derived molecules and estrogen receptor signaling pathways have been described for both associations. Accordingly, we review and discuss the general molecular mechanisms underlying estrogen metabolism, focusing on the hormones and receptors involved.

Molecular mechanism underlying estrogen metabolism Estrogens are steroid hormones produced in the ovaries, adrenal glands, and placenta during pregnancy. The hypothalamus secretes gonadotropin-releasing hormone $(\mathrm{GnRH})$, which stimulates the anterior pituitary to release follicle-stimulating hormone (FSH) and luteinizing hormone (LH). FSH and LH induce the production of estrogen in the form of estradiol and estrone by the ovaries. These estrogens bind to ERs in target tissues of the breast, uterus, brain, bone, liver, and heart [14]. When the estrogen molecule binds to its receptor, a conformational change in the ER permits its interaction with a specific regulatory sequence of the ER gene (estrogen responsive element), inducing the transcription of this target coding sequence. The resulting ER protein promotes changes in the cell according to tissue type and underlying conditions. The cycle is completed when high levels of estrogen in the blood send negative feedback to the hypothalamus to suppress the release of GnRH [14].

By the 1950s, most of the basic actions of the estrogenic hormones were recognized, such as their stimulation on the growth and function of tissues of the female reproductive tract. However, the biochemical processes involved were not entirely clear [15]. The generally accepted hypothesis was that the 17-hydroxyl group of estradiol underwent enzymatic oxidation from a cholesterol molecule using one coenzyme $(\mathrm{NADH})$, and the resulting estrone was reduced using another (NADPH) [15]. The identification of the ER provided a mechanism to describe the target site specificity of estrogen action in the uterus, vagina, pituitary gland, and breast tissue [16]. Most importantly, a test was established to predict the outcome of antihormonal therapy in breast cancer, and a target was identified to develop new drugs for the treatment and prevention of breast cancer [16].

\section{ERs and action of estrogen}

Nuclear hormone receptors belong to a family of hormoneactivated transcription factors that can initiate or enhance the transcription of genes containing specific hormone response elements [17]. The human ER, which belongs to this family, was cloned and sequenced from MCF-7 human breast cancer cells [17]. The human ER locus is located on chromosome $6 \mathrm{q}$ sub-band 25.1 [18] and the mouse ER is located on chromosome $10[19,20]$. The ER consists of 595 amino acids with a molecular mass of $66 \mathrm{kDa}$ and includes six functional domains [20-22]; two of the domains are highly conserved among the members of the nuclear hormone receptor superfamily [20-22]. Two zinc fingers at the DNA-binding domain (DBD) of ER mediate receptor binding to hormone-response elements in the promoter regions of hormone-responsive genes. The hormone-binding domain (HBD), located at the ER C terminus, exhibits two regions of sequence homology with other hormone receptors. These regions confer hormone specificity and selectivity to ER [22-26].

More recently, another sequence belonging to the nuclear hormone receptor superfamily was cloned from a rat prostate cDNA library $[27,28]$. This sequence was named ER $\beta$ (as opposed to ER $\alpha$ ). ER $\beta$ contains 485 amino acid residues and has a molecular weight of $54.2 \mathrm{kDa}$ (Figure 1). There is a high homology between ER $\alpha$ and ER $\beta$, mainly in the DBD (95\%) and the HBD (55\%), and both proteins bind estrogen with high affinity, bestowing functional homology. The latter has been determined by the activation of transcription of a vitellogenin A2, an estrogen-response element (ERE)-containing reporter plasmid in the presence and absence of estrogen [20,27].

The mechanism of target site specificity and selectivity seen with anti-estrogens, such as raloxifene, could be explained by the existence of two different ERs [29]. The receptor-specific regions are probably responsible for the differences seen between $E R \alpha$ and $E R \beta$, in spite of the high homology in the conserved regions of both ERs [20].

Estrogen diffuses through the plasma membrane of cells where it binds to the ER. Once estrogen binds to the inactive ER, the receptor is activated, a conformational change and homodimerization occurs, and two receptorligand monomers dimerize and bind to the ERE. Once bound to the ERE, the ER uses activation functions (AFs) (AF-1 and $\mathrm{AF}-2)$ to stimulate transcription from

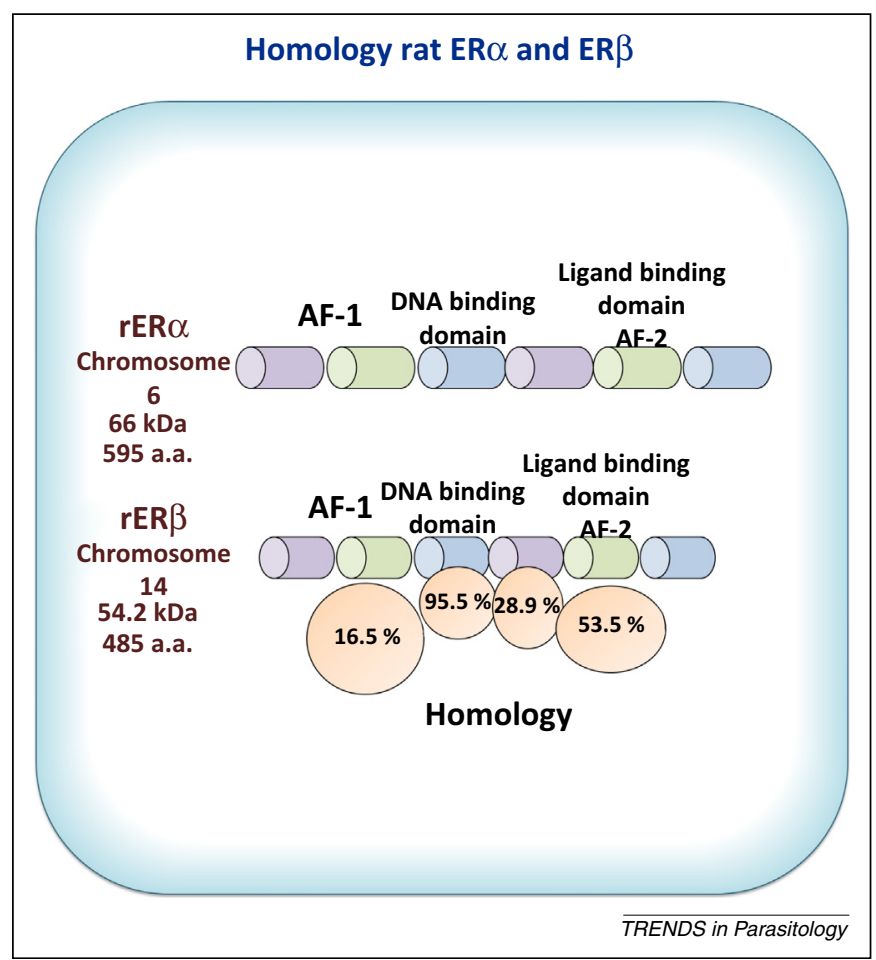

Figure 1. Comparison of the rat ( $r) \mathrm{ER} \alpha$ and $\mathrm{rER} \beta$ proteins and percent amino acid homology in the functional regions (Adapted from [20]). Abbreviation: ER, estrogen receptor. 
the promoter $[20,30]$. The ER contains two functional domain areas called AFs: AF-1 is located in the N-terminal region of the $\mathrm{ER}$ and $\mathrm{AF}-2$ is located in the $\mathrm{C}$-terminal region in the ligand-binding domain (LBD) of the ER. These are synergistic when the ER is activated by estrogen. Using mammalian cells, it was shown that the AF-1 and AF-2 regions, when expressed as separate polypeptides, functionally interact in response to estrogen and anti-estrogens, thus suggesting that estrogen binding to the ER facilitates a conformational change that brings $\mathrm{AF}-1$ and $\mathrm{AF}-2$ in direct association with one another, leading to synergy that results in transcriptional activation. These findings explained mechanistically the role of the two AFs in mediating hormone-regulated transcription $[20,28]$. The EREs are constituted by 13-bp palindromic sequences upstream the transcriptional start site. Binding of the ER to the corresponding ERE enhances the transcriptional rate of the gene in the target tissue, for example, breast [20,30].

ER mediates the biological effects of estrogens in a variety of target tissues. Ligand binding to ER stimulates gene transcription via interaction with EREs. ERs are known to mediate important physiological functions, such as reproduction, metabolism, maintenance of bone density and growth of estrogen-responsive tumors, including breast and endometrial cancers. Estrogens are also known to have a mitogenic effect in estrogen-responsive cells [31]. Progress in the regulation of the ER in breast cancer and anti-estrogen therapy has been reviewed [20].

\section{S. haematobium-associated bladder cancer}

SCC is a malignant, poorly differentiated neoplasm. SCC is the common form of bladder cancer in rural Africa where $S$. haematobium is prevalent $[32,33]$. By contrast, the majority of bladder cancer in developing countries and regions not endemic for urogenital schistosomiasis is transitional cell carcinoma (TCC), which arises from the transitional epithelium lining of the bladder. The parasite eggs trapped in the bladder wall release antigens and other metabolites (presumably evolved to expedite egress to the urine, and hence to the external environment). The phenomenon leads to hematuria and to chronic inflammation, in turn increasing the risk of SCC of the bladder. The epidemiological association between SCC of the bladder with schistosomiasis haematobia is based both on case control studies and on the correlation of bladder cancer incidence with prevalence of $S$. haematobium infection within diverse geographic locations. The incidence of urogenital schistosomiasis-associated SCC is estimated in 3-4 cases per 100000 [34]. Schistosomiasis haematobia is a chronic infection. The adult, egg-producing schistosomes live for many years, re-infections frequently occur, and schistosomiasis associated bladder SCC appears relatively early, often by the mid-decades of life (TCC usually presents in the later decades of life). In its recent monograph, IARC confirmed that chronic infection with S. haematobium causes cancer of the urinary bladder [8].

While addressing schistosomiasis-induced hypogonadism in patients infected with $S$. haematobium and $S$. mansoni, Botelho et al. observed a noteworthy elevation in serum levels of estradiol, whereas those of LH and FSH remained normal, and hypothesized that the excess estradiol could be external to the host [13]. In fact, we found that the molecule responsible for the effect was an $S$. haematobium-derived estradiol-like molecule that is an antagonist of estradiol and thus repressed the transcriptional activity of the ER. Moreover, new estrogenic molecules were identified in $S$. haematobium total antigen as well as in the serum of infected individuals with this parasitic disease that seem to be produced by this parasite [35]. ER transcriptional activity was suppressed in urothelial cells and ER expression was also suppressed in the bladders of mice in response to $S$. haematobium [36].

Estrogenic molecules present in the egg extract of $S$. haematobium were also identified and characterized by liquid chromatography-mass spectroscopy (LC-MS). The majority of these compounds are catechol estrogens [1]. Catechol estrogens are formed by hydroxylation on the steroid aromatic ring A. Hydroxylation of both C-2 and C-3 on a steroid ring was apparent and suffered further oxidation into an estradiol-2,3-quinone. The genotoxic effects of estrogen metabolites might be attributed to oxidation of catechol estrogens to quinones, followed by redox cycling and formation of reactive oxygen species that in turn react with DNA [37,38] (Figure 2).

Given the context of the unarguable link between $S$. haematobium infection and bladder cancer, the presence of putative carcinogenic molecules in $S$. haematobium eggs hopefully may have practical consequences for new approaches to disease control [1,35]. Metabolism of estrogens and the production of depurinating estrogen-DNA adducts can be implicated in a pathway underlying $S$. haematobium-promoted host cell DNA damage, leading eventually to cell transformation. The carcinogenic effect of this estrogen-DNA adductmediated pathway could explain the link between chronic schistosomiasis haematobia and SCC of the bladder [1]. We anticipate that these findings will contribute to understanding how schistosomiasis haematobia leads to SCC of the bladder.

\section{Schistosoma haematobium and infertility}

Infertility is a common medical condition, affecting one in six couples (15-20\%) worldwide [39]. Human and animal models have unraveled an association between estrogen insufficiency with abnormal spermatogenesis and male infertility [40-42]. Animal models reveal that ER $\alpha$ knockout $(\mathrm{ER} \alpha \mathrm{KO})$ and double $\mathrm{ER} \alpha / \mathrm{ER} \beta$ knockout $(\mathrm{ER} \alpha / \beta \mathrm{KO})$ mice are infertile from puberty, and display atrophy of the testes and seminiferous tubule dysmorphogenesis, likely to lead to decreased spermatogenesis and sperm motility [43]. By contrast, ER $\beta$ knockout (ER $\beta K O$ ) mice are fertile and have no apparent reproductive alterations or morphologic changes $[40,43]$.

Hormonal disturbances in women with FGS may be linked to infertility and suboptimal fecundity [11,44]. Recently, estrogen-like metabolites were detected by LC-MS in urine of S. haematobium-infected women. These metabolites are similar to those identified previously in the adult worm and egg stages of S. haematobium [1]. The presence of estrogen-like metabolites during FGS was statistically associated with self-reported infertility [11]. These electrophilic compounds can react with DNA 


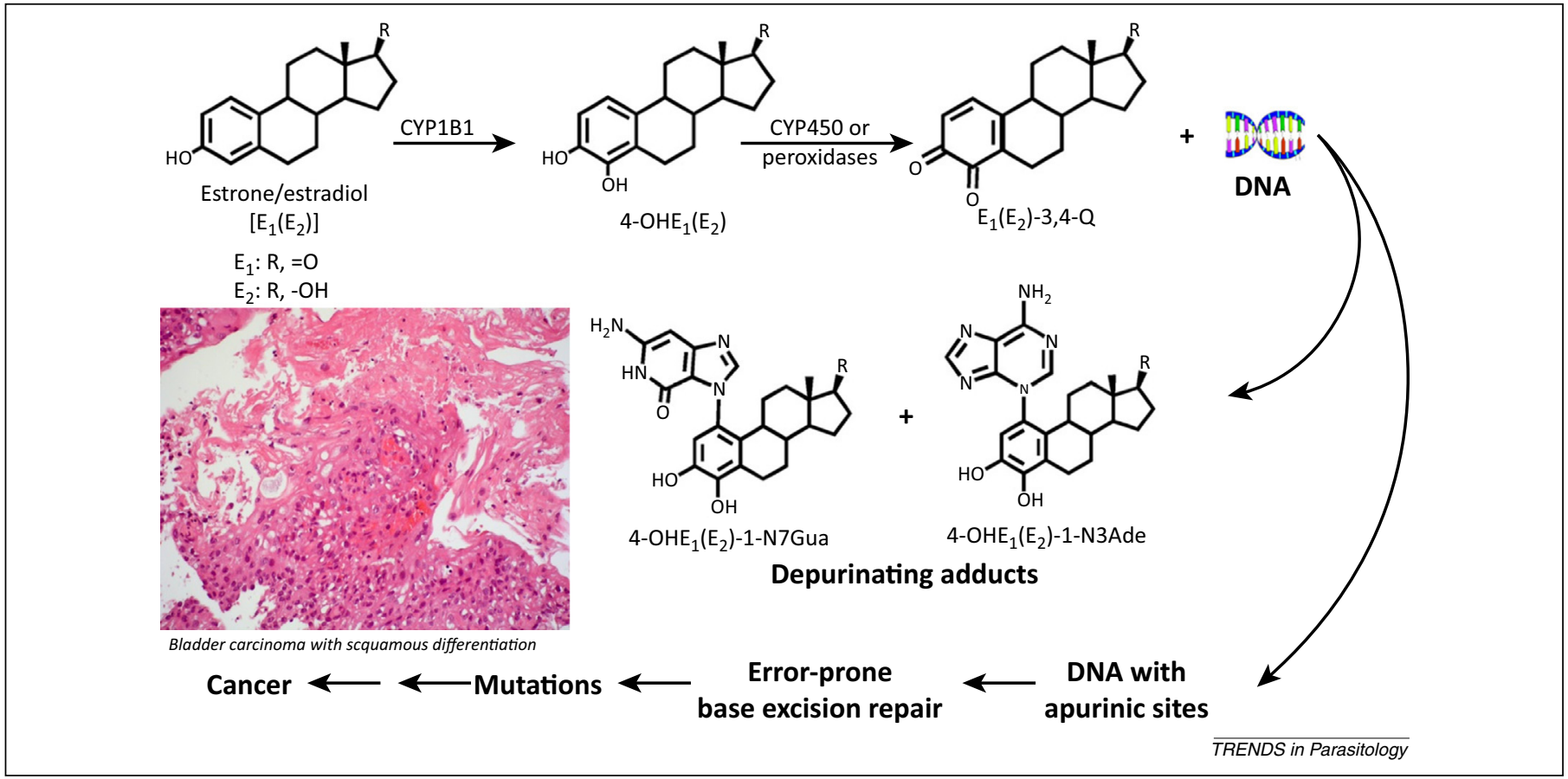

Figure 2. Major metabolic pathways in cancer initiation influenced by estrogens. The formation of catechol estrogens, that is, 2-hydroxy(OH)E1(E2) and 4-OHE1(E2) can lead through oxidation to semiquinones and quinones, for example, E1(E2)-3,4Q that eventually react with DNA to form depurinating adducts. Error-prone repair of the apurinic sites may lead to mutations that can initiate bladder carcinoma with squamous differentiation (microphotograph). (Adapted from [40]).

to form depurinating adducts. It is not inconceivable that apurinic sites in chromosomal DNA that result from this reaction generate mutations that might underlie infertility $[11,44]$.

\section{Concluding remarks and future perspectives}

Studies are necessary to identify and characterize production of these estradiol-like moieties in schistosomes and ascertain the functions of the hormone in the developmental cycle of the blood fluke. Indeed, blocking the binding of this molecule to its receptor, with the use of antihormonal therapy such as ICI 182,780, a potent anti-estrogen with the ability to inhibit and downregulate ER [45], could be explored as a complementary therapy to schistosomiasis.

A functional estrogen transmembrane receptor, G protein-coupled receptor (GPR)30, modulates both rapid nongenomic events and genomic transcriptional events of estrogen. GPR30 promotes the progress of estrogen-related tumors through mitogen-activated protein kinase (MAPK) signaling pathways. Effects mediated by GPR30 are maintained when classic ERs are absent or blocked. In addition, GPR30 is involved in drug resistance, which is often occurring during cancer treatment. Hence, simultaneous blocking both GPR30 and classic ERs may be a better strategy for the treatment of schistosomiasis-related cancer and infertility [46].

It will be informative to assess the specific effects of the estrogenic molecules identified in the lysates of schistosomes and to evaluate activities of specific catechol estrogens identified in the schistosome eggs, either by using catechol estrogens purified from eggs of $S$. haematobium and/or synthetic versions of these putative carcinogens. In addition, given that the genome and transcriptome of eggs, female and male adult worms of $S$. haematobium are available, studies utilizing RNAi to silence components of estrogen catabolism pathways such as schistosome estradiol 17- $\beta$ dehydrogenase and other candidate genes should be informative [47-49].

\section{Acknowledgments}

UMIB is funded by National Funds through FCT-Foundation for Science and Technology, under the "Fcomp-01-0124-FEDER-015893" project.

\section{References}

1 Botelho, M.C. et al. (2013) Tumour-like phenotypes in urothelial cells after exposure to antigens from eggs of Schistosoma haematobium: an oestrogen-DNA adducts mediated pathway? Int. J. Parasitol. 43, 17-26

2 van der Werf, M.J. et al. (2003) Quantification of clinical morbidity associated with schistosome infection in sub-Saharan Africa. Acta Trop. 86, 125-139

3 Hotez, P.J. et al. (2009) Africa's 32 cents solution for HIV/AIDS. PLoS Negl. Trop. Dis. 3, e430

4 King, C.H. (2010) Parasites and poverty: the case of schistosomiasis. Acta Trop. 113, 95-104

5 Hodder, S.L. et al. (2000) Predisposition to urinary tract epithelial metaplasia in Schistosoma haematobium infection. Am. J. Trop. Med. Hyg. 63, 133-138

6 Parkin, D.M. (2006) The global health burden of infection-associated cancers in the year 2002. Int. J. Cancer 118, 3030-3044

7 Bouvard, V. et al. (2009) A review of human carcinogens - Part B: biological agents. Lancet Oncol. 10, 321-322

8 IARC, Biological agents. (2012). Volume 100 B. A review of human carcinogens. IARC monographs on the evaluation of carcinogenic risks to humans/WHO, IARC. 100(Pt B), 1-441

9 Feldmeier, H. et al. (1994) Female genital schistosomiasis as a riskfactor for the transmission of HIV. Int. J. STD AIDS 5, 368-372

10 Kjetland, E.F. et al. (2006) Association between genital schistosomiasis and HIV in rural Zimbabwean women. AIDS 20, 593-600

11 Jourdan, P.M. et al. (2011) Increased vascularity in cervicovaginal mucosa with Schistosoma haematobium infection. PLoS Negl. Trop. Dis. 5, e1170

12 Santos, J. et al. (2014) Urinary estrogen metabolites and self-reported infertility in women infected with Schistosoma haematobium. PLoS ONE 9, e96774 
13 Botelho, M.C. et al. (2009) Schistosoma haematobium and Schistosomiasis mansoni: production of an estradiol-related compound detected by ELISA. Exp. Parasitol. 122, 250-253

14 Jensen, E.V. (2005) The contribution of "alternative approaches" to understanding steroid hormone action. Mol. Endocrinol. 19, 14391442

15 Jensen, E.V. and Jordan, V.C. (2003) The estrogen receptor: a model for molecular medicine. Clin. Cancer Res. 9, 1980-1989

16 Greene, G.L. et al. (1986) Sequence and expression of human estrogen receptor complementary DNA. Science 231, 1150-1154

17 Menasce, L.P. et al. (1993) Localization of the estrogen receptor locus (ESR) to chromosome $6 \mathrm{q} 25.1$ by FISH and a simple post-FISH banding technique. Genomics 17, 263-265

18 Sluyser, M. et al. (1988) Assignment of estradiol receptor gene to mouse chromosome 10. J. Steroid Biochem. 31, 757-761

19 MacGregor, J.I. and Jordan, V.C. (1998) Basic guide to the mechanisms of antiestrogen action. Pharmacol. Rev. 50, 151-196

20 Kumar, V. et al. (1986) Localisation of the oestradiol-binding and putative DNA-binding domains of the human oestrogen receptor. EMBO J. 5, 2231-2236

21 Kumar, V. et al. (1987) Functional domains of the human estrogen receptor. Cell 51, 941-951

22 Krust, A. et al. (1986) The chicken oestrogen receptor sequence: homology with v-erbA and the human oestrogen and glucocorticoid receptors. EMBO J. 5, 891-897

23 Kumar, V. and Chambon, P. (1988) The estrogen receptor binds tightly to its responsive element as a ligand-induced homodimer. Cell 55, 145-156

24 Carson-Jurica, M.A. et al. (1990) Steroid receptor family: structure and functions. Endocr. Rev. 11, 201-220

25 Ortí, E. et al. (1992) Phosphorylation of steroid hormone receptors. Endocr. Rev. 13, 105-128

26 Kuiper, G.G. et al. (1996) Cloning of a novel receptor expressed in rat prostate and ovary. Proc. Natl. Acad. Sci. U.S.A. 93, 5925-5930

27 Katzenellenbogen, B.S. and Korach, K.S. (1997) A new actor in the estrogen receptor drama - enter ER-beta. Endocrinology 138, 861-862

28 Kuiper, G.G. et al. (1997) Comparison of the ligand binding specificity and transcript tissue distribution of estrogen receptors alpha and beta. Endocrinology 138, 863-870

29 Bai, Z. and Gust, R. (2009) Breast cancer, estrogen receptor and ligands. Arch. Pharm. (Weinheim) 342, 133-149

$30 \mathrm{Yu}$, K.D. et al. (2010) A systematic review of the relationship between polymorphic sites in the estrogen receptor-beta (ESR2) gene and breast cancer risk. Breast Cancer Res. Treat 126, 37-45

31 Botelho, M.C. et al. (2011) Targeting molecular signaling pathways of Schistosoma haematobium infection in bladder cancer. Virulence 2 267-279

32 Mostafa, M.H. et al. (1999) Relationship between schistosomiasis and bladder cancer. Clin. Microbiol. Rev. 12, 97-111
33 Zhong, X. et al. (2013) Hypermethylation of genes detected in urine from Ghanaian adults with bladder pathology associated with Schistosoma haematobium infection. PLoS ONE 8, e59089

34 Shiff, C. et al. (2006) Ultrasound verification of bladder damage is associated with known biomarkers of bladder cancer in adults chronically infected with Schistosoma haematobium in Ghana. Trans. R. Soc. Trop. Med. Hyg. 100, 847-854

35 Botelho, M.C. et al. (2010) Schistosoma haematobium: identification of new estrogenic molecules with estradiol antagonistic activity and ability to inactivate estrogen receptor in mammalian cells. Exp. Parasitol. 126, 526-535

36 Botelho, M.C. et al. (2012) Inactivation of estrogen receptor by Schistosoma haematobium total antigen in bladder urothelial cells. Oncol. Rep. 27, 356-362

37 Cavalieri, E.L. et al. (1997) Molecular origin of cancer: catechol estrogen-3,4-quinones as endogenous tumor initiators. Proc. Natl. Acad. Sci. U.S.A. 94, 10937-10942

38 Cavalieri, E.L. and Rogan, E.G. (2011) Unbalanced metabolism of endogenous estrogens in the etiology and prevention of human cancer. J. Steroid Biochem. Mol. Biol. 125, 169-180

39 Sousa, M. et al. (2012) Outcomes of human blastocyst transfer after slow-freezing using sequential culture: a clinical report. Arch. Gynecol. Obstet. 285, 1473-1478

40 Saunders, P.T. (2005) Does estrogen receptor beta play a significant role in human reproduction? Trends Endocrinol. Metab. 16, 222-227

41 Hess, R.A. (2003) Estrogen in the adult male reproductive tract: a review. Reprod. Biol. Endocrinol. 9, 1-52

42 Khattri, A. et al. (2009) Estrogen receptor beta gene mutations in Indian infertile men. Mol. Hum. Reprod. 15, 513-520

43 Meng, J. et al. (2013) Influence of the XbaI polymorphism in the estrogen receptor- $\alpha$ gene on human spermatogenic defects. Genet. Mol. Res. 12, 1808-1815

44 Botelho, M.C. and Sousa, M. (2014) New biomarkers to fight urogenital schistosomiasis: a major neglected tropical disease. Biomark. Med. 8, 1061-1063

45 Guerreiro, S. et al. (2007) Distinct modulation of alkaline phosphatase isoenzymes by 17 beta-estradiol and xanthohumol in breast cancer MCF-7 cells. Clin. Biochem. 40, 268-273

46 Wang, D. et al. (2010) G protein-coupled receptor 30 in tumor development. Endocrine 38, 29-37

47 Rinaldi, G. et al. (2011) Genetic manipulation of Schistosoma haematobium, the neglected schistosome. PLoS Negl. Trop. Dis. 5, e1348

48 Duvoisin, R. et al. (2012) Human U6 promoter drives stronger shRNA activity than its schistosome orthologue in Schistosoma mansoni and human fibrosarcoma cells. Transgenic Res. 21, 511-521

49 Young, N.D. et al. (2012) Whole-genome sequence of Schistosoma haematobium. Nat. Genet. 44, 221-225 